Après le discours d'ouverture pronouncé par M. Janne, Directeur de l'Institut Solvay, en présence du Ministre des Colonies, M. Buisseret, deux commissions se sont constituées, l'une consacrée aux problèmes économiques, l'autre aux problèmes sociaux et juridiques.

Successivement ont été exposés et discutés les aspects sociaux, économiques, juridiques, éducatifs et techniques des problèmes posés par l'évolution consécutive à la présence des Occidentaux dans l'Afrique au sud du Sahara : abandon du milieu rural, développement des centres urbains et accroissement de leur population, coexistence de deux économies, traditionnelle et moderne, transformation de la propriété foncière qui, de collective, tend à devenir individuelle, principalement.

Le rôle joué dans la conjoncture actuelle par le Fonds du Bien-Être indigène au Congo Belge et au Ruanda-Urundi, par la création de paysannats et l'organisation d'un artisanat, les effets de l'enseignement professionnel, ceux de l'introduction de cultures nouvelles, les conséquences de l'extension du mouvement coopératif, ont été examinés dans leurs répercussions sociales. Les commissions ayant présenté leurs rapports, des conclusions générales ont été discutées; et le discours de clôture a été prononcé par M. Doucy, Secrétaire de l'Institut Solvay.

Le colloque a conclu qu'aucune intervention européenne ne devait être envisagée, dans le domaine économique, juridique, éducatif, technique, à plus forte raison dans le domaine social, sans l'accord des indigènes et sans que des études préalables approfondies, ethnographiques, ethnologiques et sociologiques, aient été poursuivies tant dans les milieux traditionnels que dans les nouveaux centres urbains qui demeurent étroitement solidaires les uns des autres.

Les travaux et les conclusions du colloque feront l'objet de publications par les soins de l'Institut Solvay.

J.-P. LEBeur

\title{
Les premiers Examens universitaires au Congo Belge
}

EN r959, pour la première fois au Congo Belge, des étudiants européens et africains ont présenté des examens universitaires à la fin de l'année académique à l'Université Lovanium, Kimuenza.

Pour ses débuts l'Université Lovanium comprenait trois années d'études distinctes: la première année de candidature en sciences naturelles et médicales (préparatoire à la Médecine et à l'Agronomie); la première année de candidature en sciences pédagogiques et la première année de candidature en sciences sociales et administratives. Les résultats de cette première année universitaire furent excellents. Au total sur 2I candidats universitaires (dont 3 Européens) on trouve trois grandes distinctions, quatre distinctions, des satisfactions et seulement deux échecs. Le jury universitaire était présidé par le chanoine Gillon, Recteur de Lovanium et spécialisé en physique nucléaire.

Plusieurs personnalités universitaires étaient venues spécialement de Belgique pour prendre place parmi le jury, notamment les représentants des quatre universités belges: MM. Gillis, recteur de l'Université de Gand; Van den Dungen, ancien recteur de l'Université Libre de Bruxelles; Delbouille, remplaçant le recteur de l'Université de Liège, et Mgr de Raeymacker, Président de l'Institut Supérieur de Philosophie et délégué par l'Université de Louvain.

Cette première expérience universitaire s'est révélée un succès et les délégués des quatre universités ont été unanimes à le reconnaître. Il est dû en partie, comme l'a souligné très justement M. Van den Dungen, aux conditions idéales dans lesquelles les étudiants travaillent: régime d'internat et petit nombre. Enfin, comme l'a déclaré Mgr de Raeymacker, 'l'on peut affirmer désormais sans crainte d'être contredit, que dès la première année l'enseignement donné à Lovanium a atteint le niveau de l'enseignement donné dans les universités belges. Hommage peut être rendu au corps professoral.' 